\title{
UV LASER DECAY OF OXYGEN-DEFICIENT CENTERS IN SILICA GLASSES
}

\author{
V. N. BAGRATASHVILI and S. I. TSYPINA \\ Science-Research Center for Technological Lasers of Rus. Ac. Sci., Troitsk, \\ Moscow Region, 142092, Russia
}

\author{
S. S. ALIMPIEV, YA. O. SIMANOVSKI and A. M. PROKHOROV \\ Institute of General Physics of Rus. Ac. Sci., ul. Vavilova 38, \\ Moscow, 117924, Russia
}

\author{
A. O. RYBALTOVSKI \\ Institute of Nuclear Physics of Moscow State University, Leninskie Gory, V-234, \\ Moscow, 119899, Russia
}

Oxygen-deficient centers decay with simultaneous formation of color centers in IR and UV grade silica glasses under $\mathrm{KrF}$ laser irradiation was studied. The nonexponential dependence of the ODC photodecay in silica glass on the UV irradiation dose (number of laser pulses) was observed. Postpulse long-time temporal behaviour of recombination luminescence and laser induced photoconductivity was analyzed for the two types of glasses in time scale up to $0.5 \mathrm{~ms}$. Long time fluorescence tail is attributed to migration of electrons through the shallow traps before recombination and recovery of ODC. The models of postpulse recombination which can explain the observed nonexponential behaviour of ODC decay (geminal and homogeneous ) are discussed. The principle part of impurities caused electron traps in the postpulse ODC recombination is shown.

\section{INTRODUCTION}

One of the most familiar defects of amorphous silicon dioxide $\left(\mathrm{a}-\mathrm{SiO}_{2}\right)$ is the oxygen-deficient center (ODC), being studied already over 30 years (References 1 and 2 and references therein). In the recent few years intense efforts have been made to study the action of a powerful UV laser radiation on silica glasses containing ODC. ${ }^{3-6}$ The presence of ODC in silica glass determine the optical properties in UV spectral region. In Reference 3 it was particularly shown that a $\mathrm{KrF}$ laser irradiation doses $0.1-1 \mathrm{~J} / \mathrm{cm}^{2}$ results in the decrease of absorption band near $248 \mathrm{~nm}$ caused by ODC decay and formation of paramagnetic $\mathrm{E}^{\prime}$ type color centers with absorption band near $215 \mathrm{~nm}$. In References 5 and 6 it was also shown that ODC photochemical decay and $\mathrm{E}^{\prime}$ center formation can have both single and two photon nature. In the 
case of two photon decay of ODC the first $5 \mathrm{eV}$ photon resonantly excites the $\mathrm{S}_{1}$ singlet state and the second provides ODC ionization:

$$
\equiv \mathrm{Si}-\mathrm{Si} \equiv(\mathrm{ODC}) \stackrel{2 \mathrm{~h} v}{\longrightarrow} \equiv \mathrm{Si} \uparrow \underset{\mid}{\mid} \mathrm{Si}-\left(\mathrm{E}^{\prime} \text { center }\right)+\overline{\mathrm{e}}
$$

At the considerable concentrations of metal impurities the single-quantum UV photodecay of ODC with formation of $E^{\prime}$ centers can also take place as a result of intraband (tunnel) electron transition from the first excited triplet $T_{1}$ state of ODC to the impurity level.

It should be noted that considerable increasing of UV irradiation doses $\left(>10^{3} \mathrm{~J} / \mathrm{cm}^{2}\right)$ can lead to $\mathrm{E}^{\prime}$ center formation in high pure glass free of ODC's, as a result of non-linear two-photon absorption in $\mathrm{SiO}_{2}{ }^{7}$

One of the most significant points that have to be clarified in ODC photochemistry is the part of reverse reactions, thermal processes and the secondary chemical reactions of $\mathrm{E}^{\prime}$ centers, which take place just after the UV irradiation. It is the very important particularly the influence of electron traps on the nature of postpulse elementary processes. Such influence was demonstrated in Reference 8 under $\gamma$-irradiation of silica glasses. It is clear that the nature, concentrations and distribution of electron traps must depend on impurity composition, silica glass production technology and sample treatment. The subject of this study is the detail analysis of ODC photodecay under UV laser irradiation and the part of reverse reactions, leading to recombination of laser ionized ODC's.

\section{EXPERIMENT}

In the experiments the samples of silica glasses of types I and IV according to the classification of Reference 9, were analyzed. The IR grade silica glass pertaining to type I contained significant up to $0.01 \%$ metal impurities. These samples had $0.2 \mathrm{~cm}^{-1}$ absorption at $248 \mathrm{~nm}$. The UV grade samples of type IV produced by Vapor Axial Deposition (VAD) technique contained poor metal but significant (up to $10^{-2} \%$ ) chlorine impurities. These VAD samples had $0.5 \mathrm{~cm}^{-1}$ absorption at $248 \mathrm{~nm}$.

Excimer $\mathrm{KrF}$ laser with $20 \mathrm{~ns}$ pulse duration and $\lambda=248 \mathrm{~nm}$ wavelength was used for sample irradiation. All samples had rectangular shape of $8 \mathrm{~mm}$ length with $4 \times 4$ cross section. The laser beam was collimated in a sample to obtain $2 \mathrm{~mm}$ spot diameter. Several experimental techniques were used in this study: UV absorption spectroscopy; electron spin resonance (ESR); laser induced fluorescence (LIF); photoionization technique. The silica glass absorption spectra before and after irradiation were recorded by Specord M40 spectrophotometer. The relative concentrations of laser-induced paramagnetic $\mathrm{E}^{\prime}$ centers were defined from absorption spectra near $215 \mathrm{~nm}$. The absolute calibration of $E^{\prime}$ centers concentration was performed with the use of ESR spectrometer RE 1306 operating at $9.4 \mathrm{GHz}$ frequency.

For the photocurrent measurements the analyzed sample was placed between two 


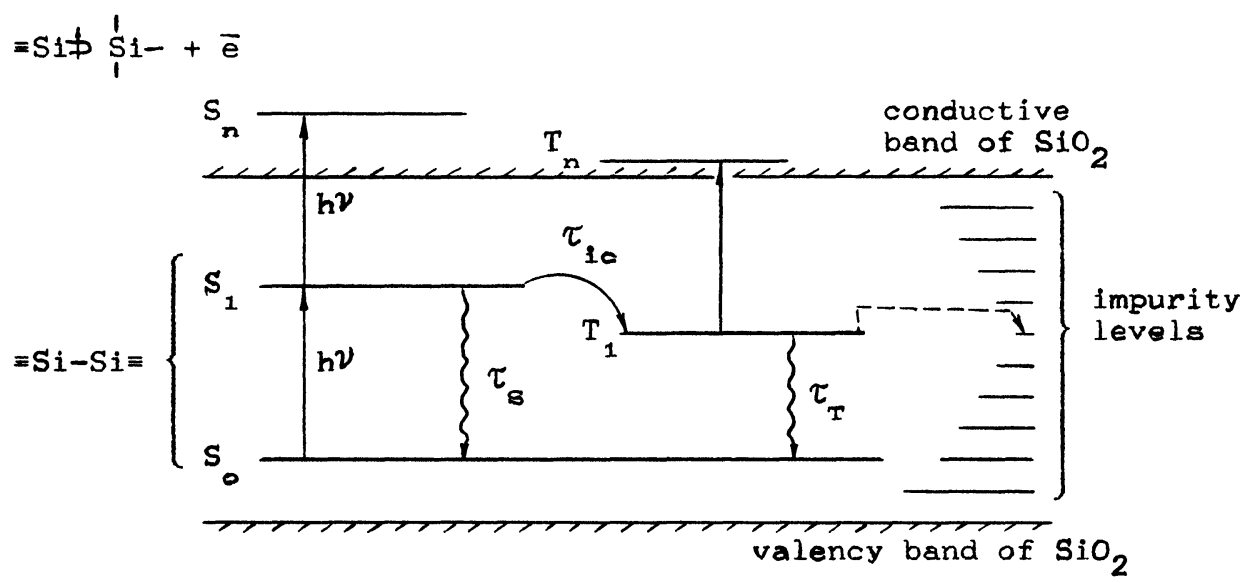

Figure 1 The energy level diagram of oxygen-deficient center in silica glass. Singlet luminescence: $\tau_{\mathrm{s}} \simeq 10^{-9}-10^{-8} \mathrm{~ns},{ }^{10} \lambda_{\max }=280 \mathrm{~nm}$; Triplet luminescence : $\tau_{\mathrm{T}} \simeq 10 \mathrm{~ms},{ }^{10} \lambda_{\max }=450 \mathrm{~nm}$; Intercombination $(\mathrm{S}-\mathrm{T})$ conversion: $\tau_{\mathrm{ic}} \simeq 14$ nc. $^{11}$

molybdenum plates. This material was chosen to diminish photoeffect signal which arises under the plates illumination by the scattered radiation. The photocurrent signal was recorded by $10 \mathrm{MHz}$ C9-8 digital oscilloscope with computer. Since the conductivity signal is proportional to the concentration and mobility of electrons in the $\mathrm{SiO}_{2}$ conduction band such measurement gives the information about the free electrons creation and trapping.

Both multichannel and single channel optical systems were used for LIF measurements. With the use of multichannel system the ODC luminescence spectrum ODC was recorded. This spectrum consist of two band near 280 and $450 \mathrm{~nm}$ associated with singlet $\mathrm{S}_{1}-\mathrm{S}_{0} \quad\left(\tau<10^{-8} \mathrm{~s}\right)$ and triplet $\mathrm{T}_{1}-\mathrm{S}_{0} \quad(\tau \simeq 10 \mathrm{~ms})$ luminescence shown in Figure 1. Single channel system due to much higher dynamic range was used to analyze the temporal behavior of recombination luminescence (RL). These measurements performed with Jobin-Yvon grating monochromator set to $\lambda=280 \mathrm{~nm}$ and PM-100 photomultiplier linked to C9-8 digitizer and computer. To measure the temperature dependence of RL signal the glass samples were placed into the copper heating cavity. The temperature could be varied from $20^{\circ} \mathrm{C}$ up to $200^{\circ} \mathrm{C}$ with $\pm 5 \%$ accuracy.

\section{RESULTS AND DISCUSSION}

\subsection{Laser-induced decay of $O D C$ and formation of paramagnetic centers}

Shown in Figure 2 is the linear absorption spectrum transformation under UV laser irradiation of silica glass. Simultaneously with UV laser-induced decreasing of ODC absorption band near $248 \mathrm{~nm}$ the increasing of absorption near $215 \mathrm{~nm}$ 


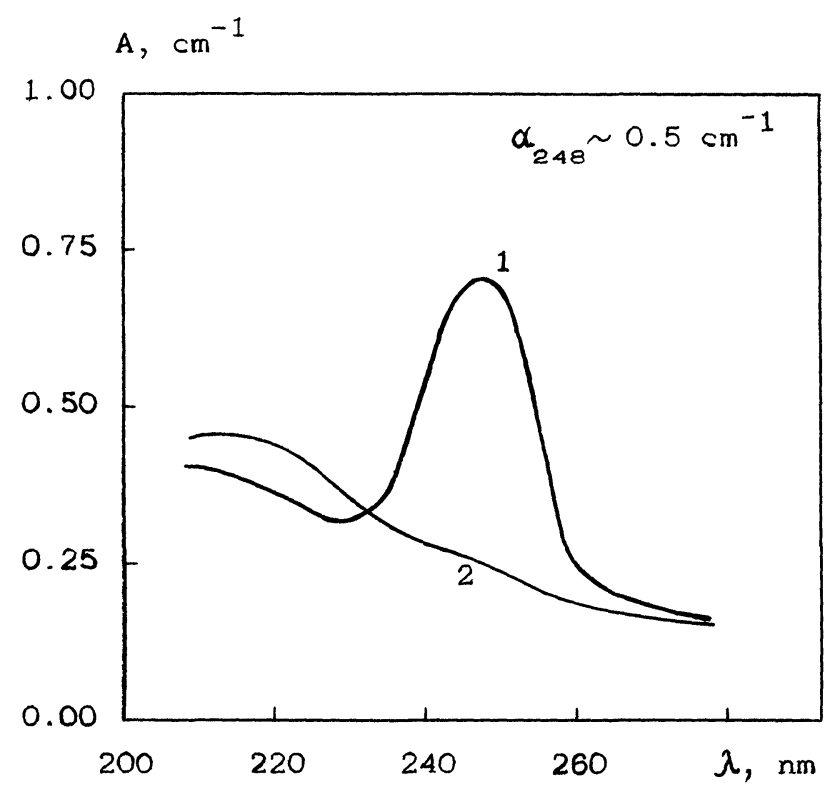

Figure 2 VAD silica glass absorption spectra before (1) and after (2) irradiation $\left(\Phi=400 \mathrm{~mJ} \mathrm{~cm}^{2}, 500\right.$ pulses).

is observed. The $215 \mathrm{~nm}$ absorption is attributed to $E^{\prime}$ center formation. This result was supported by the direct ESR measurement of $\mathrm{E}^{\prime}$ centers concentration in the irradiated samples.

The parameters of the g-tensor obtained from ESR spectra for $\mathrm{E}^{\prime}(\mathrm{Si})$-center $\left(\mathrm{g}_{\|}=2.0017\right.$ and $\left.\mathrm{g}_{\perp}=2.0003\right)$ coincide with those of the so-called $\mathrm{E}_{\gamma}^{\prime}$-center, ${ }^{2}$ i.e. the structure of $\mathrm{E}^{\prime}$ center is identical with the axisymmetrically relaxed oxygen

vacancy model ( $\equiv \mathrm{Si} \uparrow \mathrm{Si}-$ ). Besides, $\mathrm{E}^{\prime}(\mathrm{Si})$-center of axial symmetry with $\mathrm{g}_{\|}=2.001$ and $\mathrm{g}_{\perp}=1.9996$ was detected in IR samples. This second type of $\mathrm{E}^{\prime}$ center is less thermally stable than $\mathrm{E}_{\gamma}^{\prime}$-center and gets annealed at $\mathrm{T} \leqslant 200^{\circ} \mathrm{C}$. The observed concentration of such centers was $1 / 3$ of that of $\mathrm{E}_{\gamma}^{\prime}(\mathrm{Si})$-centers.

Figure 3 shows the laser fluence dependences of $E^{\prime}$ centers concentrations measured by ESR technique as well as the variation of the ODC concentration calculated from the absorption measurements at $248 \mathrm{~nm}$ for VAD and IR samples. The calculations were made with use of ODC bandwidth value $E_{248}=0.4 \mathrm{eV}$ and oscillator force $\mathrm{f}=0.2 .{ }^{10}$ In Figure 3 one can see the good correlation between the decrease of ODC concentration $\left(\Delta N_{\mathrm{ODC}}\right)$ and the amount of induced $\mathrm{E}^{\prime}$-centers $\left(N_{\mathrm{E}^{\prime}}\right)$, i.e. $\Delta N_{\mathrm{ODC}} \simeq N_{\mathrm{E}^{\prime}}$ for the IR silica glass. For the sample irradiated by 1 pulse at $\Phi=100 \mathrm{~mJ} / \mathrm{cm}^{2}$ the calculated value $\Delta N_{\mathrm{ODC}} \simeq(4.1 \pm 0.5) \times 10^{14} \mathrm{~cm}^{-3}$ is in a good agreement with concentration of induced $\mathrm{E}^{\prime}(\mathrm{Si})$-center $N_{\mathrm{E}^{\prime}} \simeq(5.2 \pm 0.5) \times 10^{14}$ $\mathrm{cm}^{-3}$. On the contrary in VAD silica glass the amount of $\mathrm{E}^{\prime}$ centers is much less 


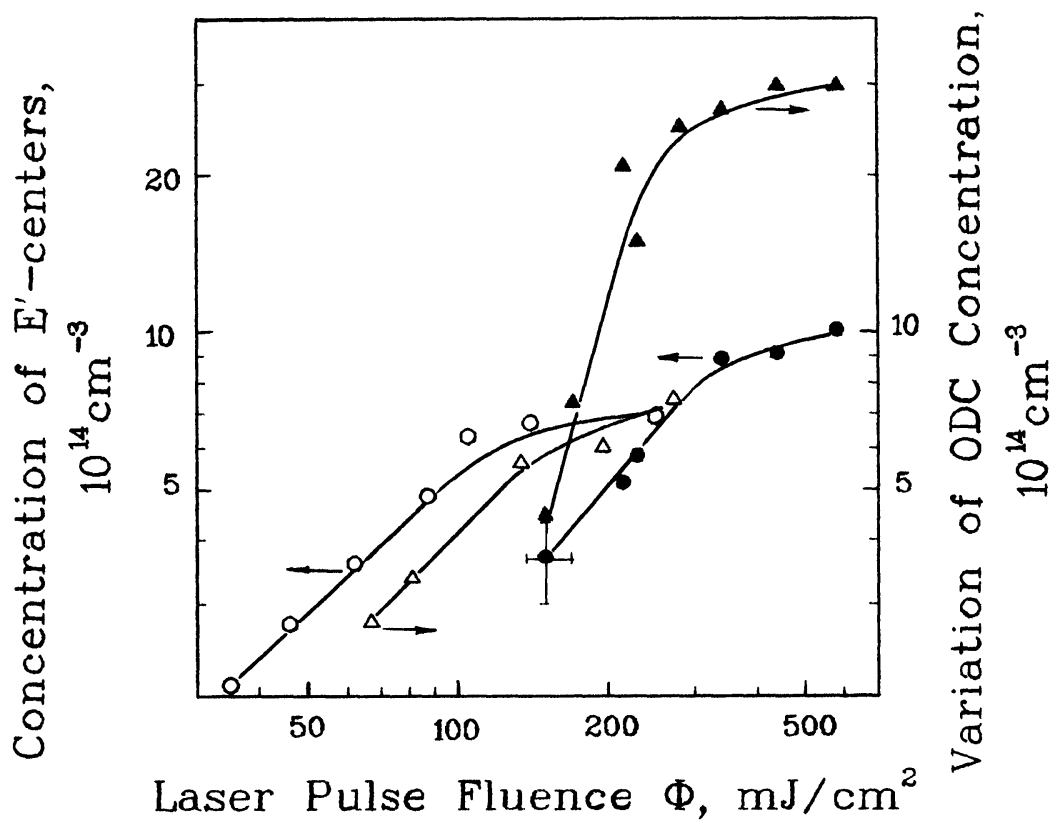

Figure 3 Reduction of ODC concentration $\left(\Delta N_{\mathrm{ODC}}\right)$ and formation of $\mathrm{E}^{\prime}(\mathrm{Si})$-centers vs laser pulse fluence in VAD $(\Delta \odot)$ and IR $(\Delta O)$ silica samples, irradiated by single pulse.

than the decrease of ODC concentration. This fact can be attributed to the reaction of chlorine containing $\mathrm{RCl}$ species with ionized ODC in silica glass of this type, which results in the disappearance of $E^{\prime}$ centers :

$$
\equiv \mathrm{Si} \hat{\$}+\mathrm{RCl} \stackrel{\mathrm{h} v}{\rightarrow} \equiv \mathrm{SiCl}+\mathrm{R}
$$

Figure 4 shows the relative concentration of $\mathrm{E}^{\prime}$ centers and decreasing of ODC absorption band in the exposed samples of IR silica glass versus annealing temperature. Good correlation of the curves is observed: the disapppearance of $E^{\prime}$ centers leads to the restoration of initial absorption level. The full restoration of ODC concentration in UV irradiated samples under annealing is very important property of ODC and can be applied as a reversible method to change the optical parameters of silica glass.

Thus, the irradiation of silica glass with high concentration of ODC $\left(N_{\mathrm{ODC}} \simeq 10^{15}-10^{16} \mathrm{~cm}^{-3}\right)$ by $\mathrm{KrF}$ laser pulses with the fluence $\Phi=0.1-1 \mathrm{~J} / \mathrm{cm}^{2}$ immediately results in the decay of $\mathrm{ODC}$ and formation of paramagnetic $\mathrm{E}^{\prime}$ centers, i.e. the ODC in this case is really the center of oxygen-vacancy type $\equiv \mathrm{Si}-\mathrm{Si} \equiv$ and the main precursor of $E^{\prime}$ centers.

The mechanism of ODC decay depends on type of silica glass, as it follows from Figure 3. The dependence of $\Delta N_{\mathrm{ODC}} / N_{0}$ versus laser fluence $\Phi$ for VAD sample can be described by square law (two photon mechanism). At the same time the analogous dependence for IR glass sample is almost linear. We suppose that it can be explained 


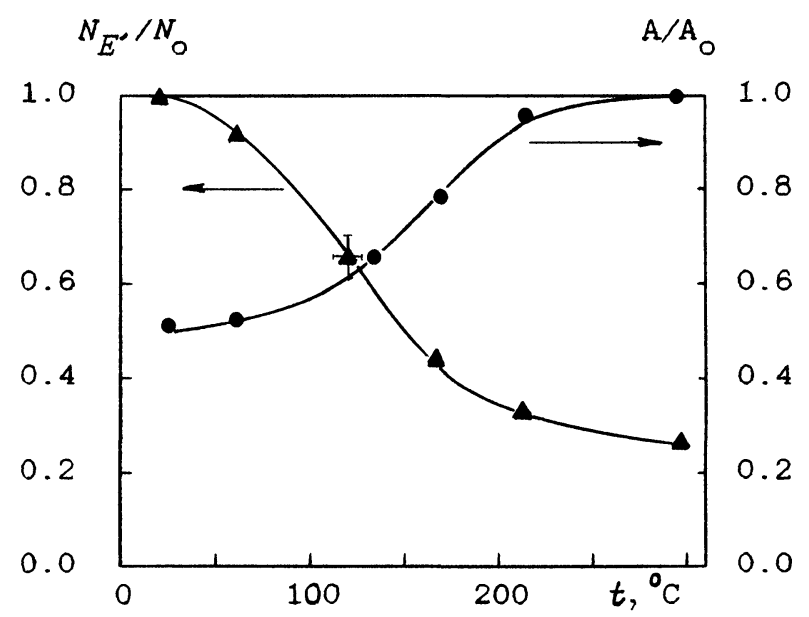

Figure 4 Restoration of initial absorption $\left(A / A_{0}\right)$ and reduction of $\mathrm{E}^{\prime}(\mathrm{Si})$-center concentration $\left(N_{\mathrm{E}^{\prime}} / N_{0}\right)$ at annealing of irradiated IR silica glass sample.

by the tunneling of electron from the first excited triplet level $\mathrm{T}_{1}$ of ODC to the impurity level. ${ }^{6}$

Finally in this section we will demonstrate the results on direct measuring of UV laser induced photocurrent in ODC containing silica samples. The duration of photocurrent pulse is almost the same as the laser pulse duration $(20 \mathrm{~ns})$. The amplitude of photocurrent pulse is proportional to the ODC concentration. The pulse-to-pulse degradation of photocurrent signal shown in Figure 5 is due to the ODC decay. We suppose that these photocurrent results are the first direct observation of UV laser photoionization (1) of ODC in silica glasses.

\subsection{Recombination luminescence}

The important information concerning the UV laser photochemistry of ODC in silica glasses was obtained by laser induced fluorescence (LIF) measurements.

We measured in details the kinetics of the singlet $\left(S_{1} \rightarrow S_{0}\right)$ fluorescence at various UV laser fluences. At very low laser fluences $\Phi<10^{-3}-10^{-4} \mathrm{~J} / \mathrm{cm}^{2}$ the duration of LIF signal is almost the same as UV laser pulse duration $20 \mathrm{~ns}$ and is not time resolved in our experiments. Intensity of fluorescence is proportional to the laser fluence $\Phi$. But if the laser fluence is higher than $10^{-2} \mathrm{~J} / \mathrm{cm}^{2}$ the kinetics of LIF signal changes drastically. Figures 6 and 7 show the singlet fluorescence $(\lambda=280 \mathrm{~nm})$ temporal behavior for IR and UV grade of silica glasses. A very long time tail (up to $5 \times 10^{-5} \mathrm{~s}$ ) is observed in both cases. The intensity of this tail measured for VAD sample is proportional to $\Phi^{2}$ and reaches saturation at $\Phi>0.2-0.3 \mathrm{~J} / \mathrm{cm}^{2}$.

We suppose that this long time fluorescence is connected with ODC decay and subsequent recombination processes. The temporal behaviour of fluorescence reflects the kinetics of the ODC recombination after the action of the UV laser pulse, since 


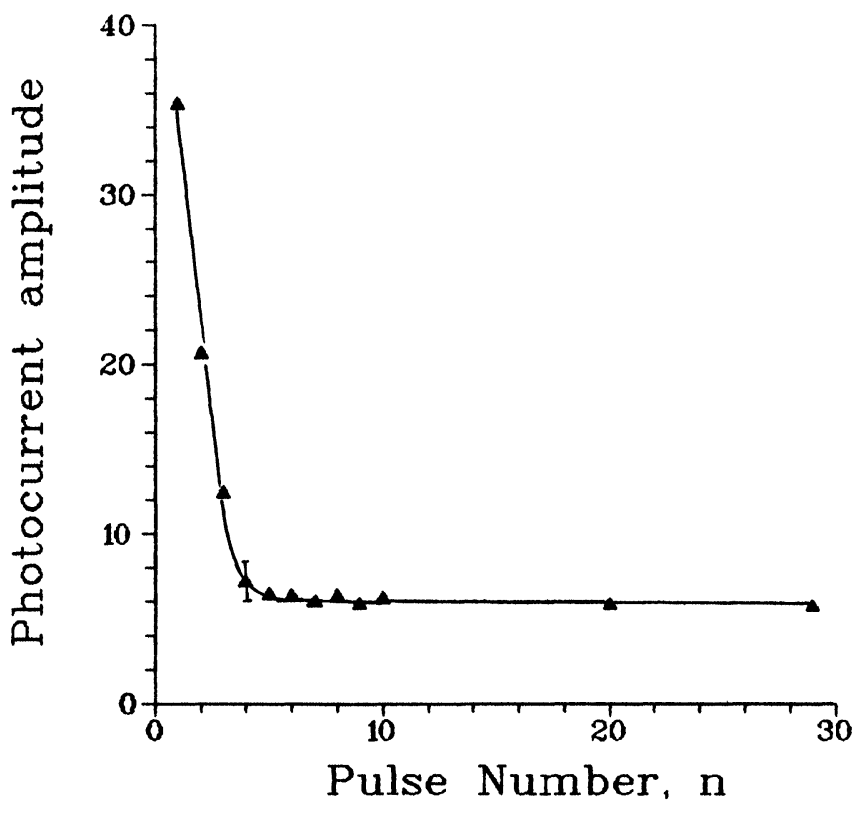

Figure 5 Amplitude of photocurrent signal for VAD sample vs number of laser pulses $\left(\Phi=0.5 \mathrm{~J} \mathrm{~cm}^{2}\right)$. The background signal at $\mathrm{n}>15-20$ is presumably due to the surface photoemission from Mo electrodes.

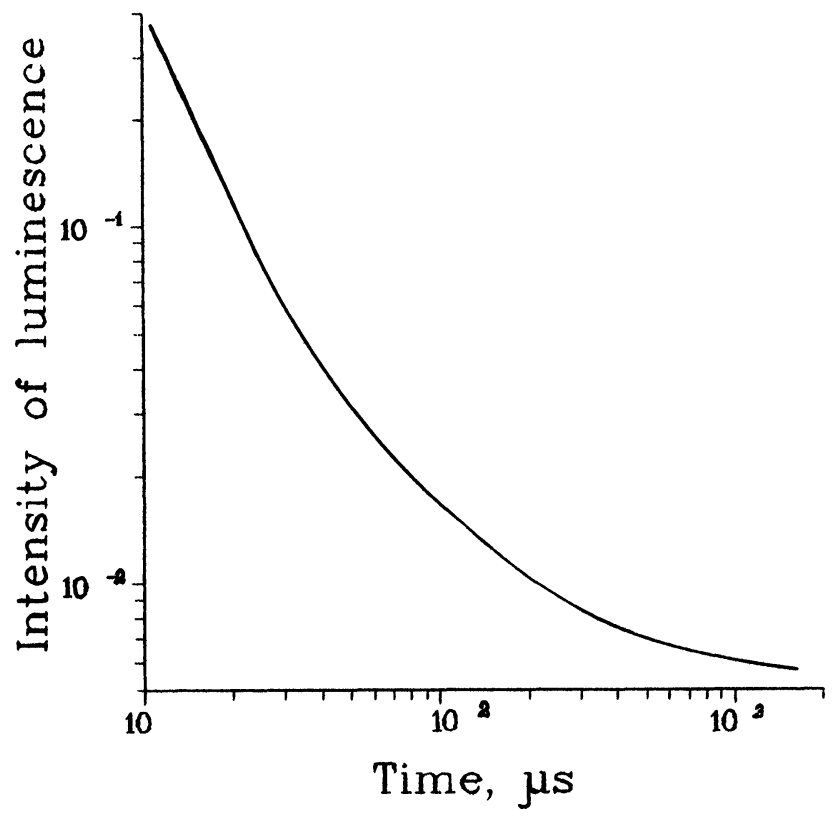

Figure 6 Kinetics of long-time postpulse recombination luminescence of VAD sample $\left(\lambda_{\text {lum }}=280 \mathrm{~nm}\right.$, $\lambda_{\text {exc }}=248 \mathrm{~nm}, \Phi_{\text {exc }}=0.5 \mathrm{~J} \mathrm{~cm}^{2}$ ). 


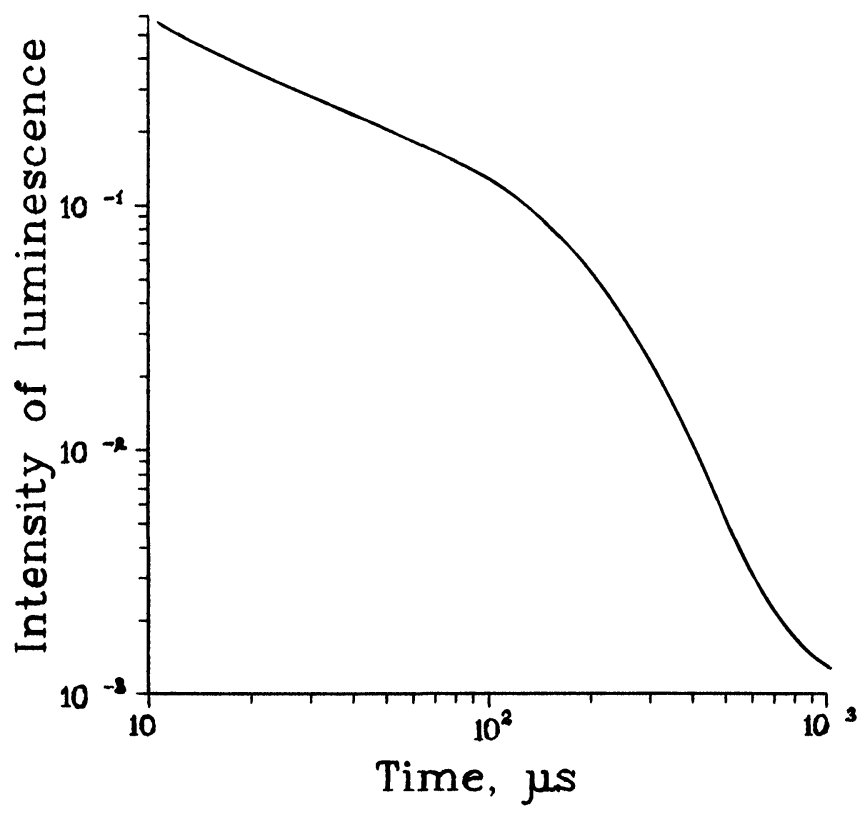

Figure 7 Kinetics of long-time postpulse recombination luminescence of IR sample $\left(\lambda_{\text {lum }}=280 \mathrm{~nm}\right.$, $\lambda_{\text {exc }}=248 \mathrm{~nm}, \Phi_{\text {exc }}=0.5 \mathrm{~J} \mathrm{~cm}^{2}$ ).

the $S_{1}$ level life time is much shorter than the duration of this recombination luminescence $(\mathrm{RL})$. As it follows from temporal behaviour analysis the RL kinetics is sensitive to the impurity content. For VAD samples the computer simulation of $\mathrm{RL}$ temporal behaviour gives the following function:

$$
\mathrm{I}_{\mathrm{RL}} \sim \mathrm{t}^{-\kappa}
$$

where $\kappa=1.58 \pm 0.05$ at $10 \mu \mathrm{s}<\mathrm{t}<50 \mu \mathrm{s}$ region and $\kappa=1.16 \pm 0.05$ at $50 \mu \mathrm{s}<\mathrm{t}<0.4 \mathrm{~ms}$. For IR silica glass sample the kinetics curve consists of two parts: the first is described by the function (3), with $\kappa=1.5 \pm 0.1$ at $10 \mu \mathrm{s}<\mathrm{t}<50 \mu \mathrm{s}$; the second is described by exponential function

$$
\mathrm{I}_{\mathbf{R L}} \sim \exp (-\mathrm{t} / \tau)
$$

where $\tau=100 \pm 10 \mu \mathrm{s}$ at $50 \mu \mathrm{s}<\mathrm{t}<1 \mathrm{~ms}$. The long RL tail is due to the electron migration after the laser ionization. The migration process first of all is determined by the nature of traps. So, the significant difference in impurity content for IR and VAD samples results in different RL kinetics (see Figures 6, 7). Secondly, the increase of the sample temperature must result in the decrease of the trapping time of electrons in shallow thermal traps. This is supported by Figure 8, which demonstrates the acceleration of recombination process after UV laser induced decay of ODC with the increase of the temperature. 


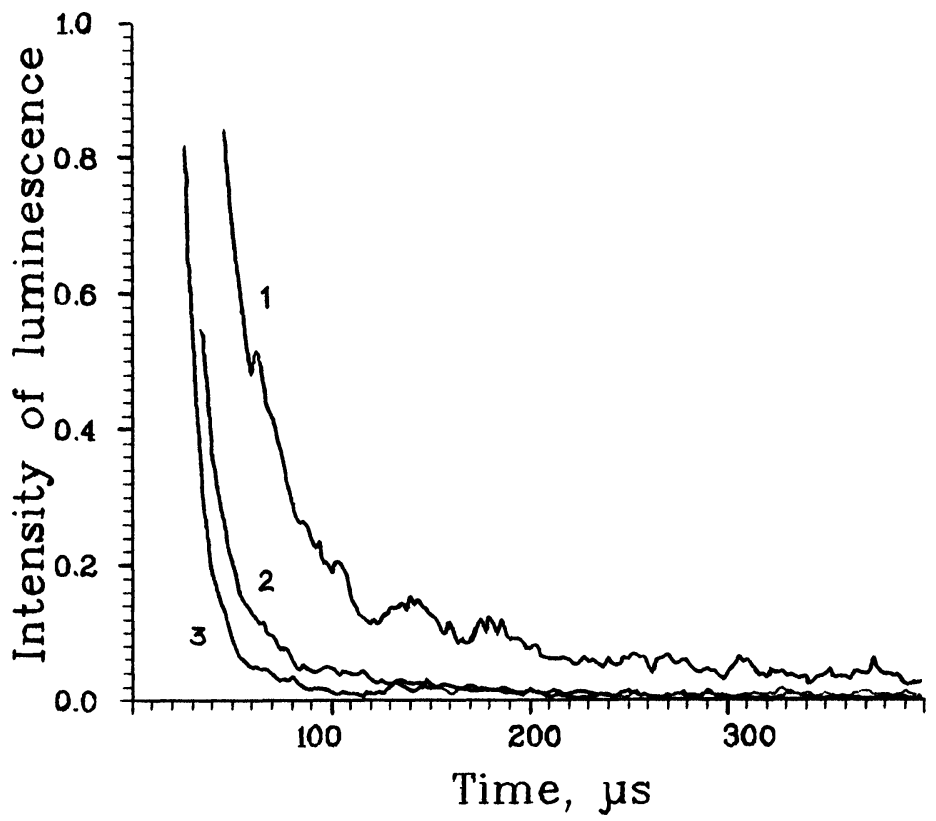

Figure 8 Oscillograms of recombination luminescence signal for VAD glass at different sample temperatures: (1), $25^{\circ} \mathrm{C} ;(2), 100^{\circ} \mathrm{C}$ and $(3), 200^{\circ} \mathrm{C}$. The curves approximation by $\mathrm{I}_{\mathrm{RL}} \sim \mathrm{t}^{-\kappa}$ function gives $\kappa$ value: $(1), 1.27 ;(2), 1.55 ;(3), 2.8$.

\subsection{Nonexponential decay of $O D C$}

The recombination processes observed by long time recombination luminescence are of great importance in the UV laser photochemistry of ODC. This is supported by the experimental results on pulse-to-pulse UV decay of ODC.

Figure 9 shows the relative concentrations of ODC $-N_{\mathrm{ODC}} / N_{0}, \mathrm{E}^{\prime}$ centers and its sum $\left(N_{\mathrm{E}^{\prime}}+N_{\mathrm{ODC}}\right) / N_{0}$ versus number of laser pulse $\mathrm{n}$ for VAD sample exposed by the laser radiation of $\Phi=400 \mathrm{~mJ} / \mathrm{cm}^{2}$ fluence. This fluence according to Figure 3 corresponds to saturation behaviour of ODC photodissociation yield $\left(\beta_{\mathrm{n}}\right)$ versus $\Phi$ dependence. The part of the ODC's decayed upon a single pulse can be determined as

$$
\beta_{\mathrm{n}}=\frac{N_{\mathrm{ODC}}(\mathrm{n})-N_{\mathrm{ODC}}(\mathrm{n}-1)}{N_{\mathrm{ODC}}(\mathrm{n})}
$$

As one can see from Figure 9 the slope of ODC decay curve decreases with the growth of the number of laser pulses indicating essentially nonexponential behaviour of $N_{\mathrm{ODC}}(\mathrm{n})$ dependence. Hence the ODC dissociation yield according with (5) drops with the growth of $n$. For the decay curve shown in Figure 9 we have $\beta_{1}=0.25$; $\beta_{10}=0.075$ and $\beta_{100}=0.01$. Similar pulse-to-pulse decreasing of ODC photodissociation yield has been also observed for other types of silica glass. ${ }^{3}$ The nonexponential decrease of ODC concentration with increase of the number of pulses 


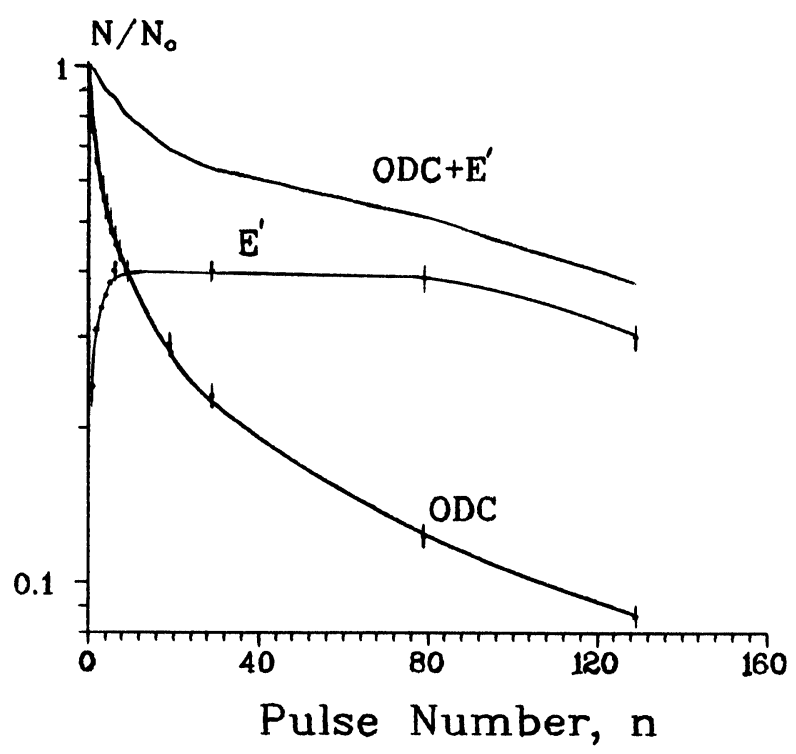

Figure 9 Relative concentrations of ODC, $\mathrm{E}^{\prime}$ centers and their sum vs laser pulse number for VAD glass with laser fluence $\Phi=400 \mathrm{~mJ} / \mathrm{cm}^{2}$.

(dose) of UV irradiation, in our opinion, is explained by the kinetics of the secondary chemical processes taking place in silica glass after ODC photodissociation and, at first, by recombination processes resulting in the restoration of ODC. Two opposite cases can be considered for the recombination processes-inhomogeneous and homogeneous.

In the inhomogeneous case the nonexponential dependence of photodissociation yield on a pulse number is naturally explained by "burning-out" of the recombination centers distinguishing by the mutual arrangement of ODC and electron traps. For the ionized ODC having large concentration of closely arranged traps, the probability of the recombination will be much less, than for ODC having a few traps in its vicinity. Therefore the final yield of ODC decay in the first case will be much more than in the second one. To develop the quantitative model, it is necessary to consider a continuous distribution function in the type of ODC surrounding, i.e. a distribution of the rate of electron catching by the traps. By varying this trap distribution function, it is easy to reach agreement with observed curve $N_{\text {ODC }}(\mathrm{n})$.

This consideration is true for the case of geminal recombination, when after ODC ionization the electron does not travel far from its ODC and so preserves its individuality. This is possible in the case of a sufficiently low concentration of ODC $N_{0}\left(\overline{\mathrm{r}}_{\mathrm{e}}\right)^{3}<1$, where $\overline{\mathrm{r}}_{\mathrm{e}}$ is the average distance passed by electron before thermalization.

In the opposite case, $N_{0}\left(\overline{\mathrm{r}}_{\mathrm{e}}\right)^{3}>1$, of very high densities of ODC a "homogeneous" situation, can be realized when the electron "forgets" the center from which it was emitted and after laser pulse the electron density is close to a constant over entire irradiated volume. In this case the geminal recombination model cannot be used, 
still the nonexponentiality can be easily explained by the filling of different types of traps.

It should be noted that a homogeneous model will feature typical behaviour even at a very big number of pulses, i.e. with $N_{\mathrm{ODC}} \ll N_{0}$. In the case of a small total concentration of traps, we have a very sharp drop of yield per pulse, associated with the saturation of total yield. At the same time, with high concentrations of traps relation $\beta(\mathrm{n})$ at $\mathrm{n} \rightarrow \infty$ must resemble the exponential one, because with small values of $\mathrm{n}$ the filling of traps can be negligible. It should be noted, however, that in the case of large "expenditures" of ODC $\left(N_{\mathrm{ODC}} \ll N_{0}\right)$, one should expect violation of the main condition of a homogeneous recombination, $N_{\mathrm{ODC}}\left(\overline{\mathrm{r}}_{\mathrm{e}}\right)^{3} \gg 1$ and transfer to inhomogeneous case. Therefore a general model can represent a combination of both geminal and homogeneous recombination.

Finally, let us come back to Figure 9. As one can see the sum of ODC and $E^{\prime}$ center relative concentrations decreases with the increase of the number of pulse. This fact cannot be explained only by the recombination processes. But it is naturally to explain by the other secondary reactions, for example, of (2) type.

\section{CONCLUSIONS}

We have shown that the UV laser induced formation of $\mathrm{E}^{\prime}$ centers in silica glasses having absorption band at $248 \mathrm{~nm}$ is the result of ionization of oxygen-deficient centers of oxygen-vacancy type $\equiv \mathrm{Si}-\mathrm{Si} \equiv$. The process of photoionization of ODC is clearly demonstrated by the direct measurement of photocurrent.

We have studied the changes of absorption band at $248 \mathrm{~nm}$ and $E^{\prime}$ center concentration versus laser pulse fluence and have demonstrated that the laser photodecay process of ODC depends on the impurity content of the silica glass.

According to this study, the important feature of the process of UV laser induced photodecay of oxygen-deficient centers in silica glass is the nonexponential dependence of ODC concentration on the number of pulses (dose) of UV radiation. The conclusion was made that the main reason of pulse-to-pulse ODC photodecay yield drop is the postpulse recombination of ionized ODC's and electrons with the formation of initial ODC's. The considered models (geminal and homogeneous) give the nonexponential dependence $N_{\mathrm{ODC}}(\mathrm{n})$. We suppose that the nature and distribution of the electron traps defined first of all by the impurity composition of silica glass plays the most important role. Our measurements of long-time post-pulse recombination luminescence shows that the different types of glass have the different kinetics of $R L$ as a result of difference in the electron trap nature.

\section{References}

1. E. P. O'Reilly and J. Robertson. Phys. Rev. B, 27, 3780 (1983).

2. D. L. Griscom. Nucl. Instr. \& Meth. Phys. Rev. B1, 229, 481 (1984).

3. V. N. Bagratashvili, A. I. Milanich, V. L. Popkov, V. K. Popov, V. A. Semchishen and S. I. Tsypina. Preprint NITSTLAN, USSR, N34 (1987); Kvantovaya Electronica, 17, 325 (1990).

4. V. N. Bagratashvili, A. O. Rybaltovski and S. I. Tsypina. Spectrochimica Acta, 46A, 665 (1990). 
5. K. Arai, H. Imai, H. Hosono, Y. Abe and H. Imagava. Appl. Phys. Lett., 53, 1981 (1988).

6. V. N. Bagratashvili, A. O. Rybaltovski and S. I. Tsypina. Preprint NITsTLAN, USSR, No. 76 (1990).

7. T. E. Tsai, D. L. Griscom and E. J. Friebele. Phys. Rev. Lett., 61, 444 (1988).

8. R. C. Hughes. Radiation Effects, 26, 225 (1975).

9. V. K. Leko and O. V. Mazurin. Properties of Silica Glass. L., Nauka, p. 135 (1985).

10. A. V. Amosov. Physics and Chemistry of Glass, 5, 624 (1979).

11. L. N. Skuja, A. N. Streletsky and A. D. Pakovich. Sol. State Comm., 50, 1069 (1984). 